\title{
Gene Suppression of the Chloride Channel 2 Suppressed TGF- $\beta 1$-Induced Proliferation, Collagen Synthesis, and Collagen Gel Contraction Mediated by Conjunctival Fibroblasts
}

\author{
Lixia Sun ${ }^{a}$ b Renzhe Cui ${ }^{a}$ Huan Meng ${ }^{a}$ Xiwen Liu ${ }^{c} \quad$ Xin Liu ${ }^{b}$ Yan Lu \\ Kun Liud Liang Jia e Yajuan Zheng ${ }^{\text {b }}$ \\ aDepartment of Ophthalmology, Yanbian University Affiliated Hospital, Yanbian University, Yanji, PR China; \\ ${ }^{\mathrm{b}}$ Department of Ophthalmology, Second Hospital of Jilin University, Jilin University, Changchun, PR China; ' ${ }^{\mathrm{Y} Y a n b i a n}$ \\ University Affiliated Hospital, Yanbian University, Yanji, PR China; ${ }^{\mathrm{d} D e p a r t m e n t}$ of Psychiatry, Yanbian Social Mental

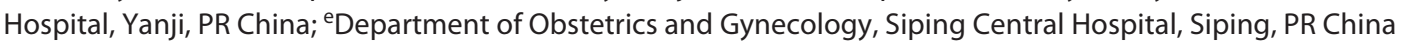

\section{Keywords}

Chloride channel 2 . Conjunctival fibroblasts - Transforming growth factor $-\beta 1 \cdot$ Wound healing

\begin{abstract}
Background: Excessive scarring of filtering blebs is the main cause of surgical failure in glaucoma. Previous studies have highlighted the role of chloride channels (CICs) in scar formation, whereas the role of $\mathrm{ClCs}$ in scarring of filtering blebs has not been studied. Objectives: The objective of this study was to investigate the effects of the chloride channel 2 (CIC2) on scar formation of filtering blebs after glaucoma filtering surgery. Methods: CIC-2 siRNA-transfected human conjunctival fibroblasts (HConFs) were cultured in type 1 collagen gels in the presence of transforming growth factor (TGF)- $\beta 1$. Collagen gel contraction was evaluated based on the gel area. 3D-cultured HConFs were treated with the $\mathrm{ClC}$ blocker NPPB in the presence of TGF- $\beta 1$, and cell proliferation collagen synthesis and contractility were measured. The expression levels of matrix metalloproteinases (MMPs) and tissue inhibitors of metalloproteinases (TIMPs) in HConFs were assessed by Western blotting and qPCR. Results: TGF- $\beta 1$ induced cell proliferation, cell cycle progression, collagen syn-
\end{abstract}

thesis, and collagen gel contraction in HConFs. TGF- $\beta 1$ increased MMP-2 and MMP-9 levels but inhibited the expression of TIMPs. NPPB and CIC-2 siRNA transfection inhibited TGF- $\beta 2$-induced cell proliferation, cell cycle progression, collagen synthesis, and collagen gel contraction, mediated by HConFs. TGF- $\beta 2$-induced increases in MMP- 2 and MMP-9 were also inhibited by NPPB and CIC-2 siRNA transfection, but TIMP expression was increased by NPPB and CIC2 siRNA transfection. Conclusions: These findings demonstrate that $\mathrm{ClC}-2 \mathrm{ClCs}$ modulate TGF- $\beta 1$-induced cell proliferation, collagen synthesis, and collagen gel contraction of HConFs by attenuating MMP-2 and MMP-9 production and by stimulating TIMP1 production. NPPB may therefore prove to be of clinical value for the inhibition of scar formation of filtering blebs.

(c) 2020 S. Karger AG, Basel

\section{Introduction}

Excessive filtering bleb scar formation after glaucoma filtration surgery is the most frequent cause of surgical failure. Human conjunctival fibroblasts (HConFs) are key cells involved in the subconjunctival wound-healing re- 
sponse, which involves the activation, proliferation, and migration of local fibroblasts as well as the synthesis of extracellular matrix components and its subsequent contraction $[1,2]$. Excessive deposition of matrix components and contraction of the subconjunctival tissue at the wound site, resulting in destruction of the normal tissue architecture, determine scar formation, and surgery failure.

Transforming growth factor (TGF)- $\beta$ is secreted during the early stages of scar formation by inflammatory cells and acts as a chemoattractant to promote fibroblast transformation to myofibroblasts, which synthesize extracellular matrix [3, 4]. Accumulation of ECM structural proteins increases bleb capsule fibrosis. Matrix metalloproteinases (MMPs) constitute a large family of enzymes that remodel ECM by degrading ECM molecules during the wound-healing process. The action of MMPs is regulated by a group of endogenous tissue inhibitors of metalloproteinases (TIMPs) [5]. Imbalance between MMPs and TIMPs may lead to excessive degradation or increased accumulation of the ECM, resulting in excessive wound healing.

Chloride channels (ClCs) are a type of permeable channel protein for chloride ions or other anions on the cell membrane, and $\mathrm{ClC}$ proteins are encoded by genes of the $\mathrm{ClC}$ family [6]. $\mathrm{ClC}-2$ is a member of the $\mathrm{ClC}$ voltagegated $\mathrm{ClC}$ superfamily and is ubiquitously expressed in organs. The effects of $\mathrm{ClC}-2$ in organs are as follows: regulation of cell volume, controlling the response to swelling, and postsynaptic responses to GABA and glycine [7, 8]. In the prevention of hyperplasia and remodeling of cerebrovascular smooth muscle cells, ClC-2 plays an important role by inhibiting cell proliferation and migration [9]. ClC-2 also modulates wound epithelialization by regulating the migratory ability of rat keratinocytes [10]. In this study, the effects of the ClC-2 ClC on cell proliferation, collagen synthesis, collagen contraction, and MMP and TIMP production by HConFs were evaluated.

\section{Materials and Methods}

Drugs

NPPB (Sigma, St. Louis, MO, USA) was prepared as a $0.4 \mathrm{M}$ stock solution in dimethyl sulfoxide (Beyotime, Jiangsu, China), stored at $<-20^{\circ} \mathrm{C}$, and protected from light until use. NPPB was diluted to $100 \mu \mathrm{M}$ in Dulbecco's modified Eagle's medium (Sigma) on the day of the experiment. The concentration of dimethyl sulfoxide in the test solutions was $0.02 \%$.

\section{Cell Culture}

HConFs were obtained from ScienCell Research Laboratories (San Diego, CA, USA), where they were isolated from hu- man conjunctiva. HConFs were characterized by their spindle morphology and immunoreactivity with antibodies to fibronectin. Cells were grown at $37^{\circ} \mathrm{C}$ in a humidified incubator with $5 \%$ $\mathrm{CO}_{2}$ in fibroblast medium (ScienCell Research Laboratories) containing $2 \%$ FBS, $100 \mathrm{U} / \mathrm{mL}$ penicillin, $100 \mu \mathrm{g} / \mathrm{mL}$ streptomycin, and fibroblast growth supplement (undisclosed formulation).

\section{Transfection of HConFs with siRNA}

To knockdown ClC-2 expression, we obtained commercially available stealth siRNA duplex oligoribonucleotides targeting the human $\mathrm{ClC}-2$ gene. The sequence was 5'-UCCUCAUGAGGAAACGCCUGCUCUU- ${ }^{\prime}$, and its corresponding complementary strand was $5^{\prime}$-AAGAGCAGGCGUUUCCUCAUGAGGA-3'. The negative control consisted of a nonsilencing scrambled sequence that did not recognize any known sequence available in the GenBank. To examine the uptake of ClC-2 siRNA by HConFs, siRNA was labeled with Alexa Fluor 488 (Invitrogen Life Technologies, Inc., Carlsbad, CA, USA) for determination of siRNA transfection efficiency. HConFs were transfected with oligonucleotides in the presence of Lipofectamine 2,000 (Invitrogen Life Technologies, Inc., Carlsbad, CA, USA) in Opti-MEMI (Invitrogen Life Technologies, Inc.) for $4 \mathrm{~h}$ at $37^{\circ} \mathrm{C}$. After removing the transfection mixture, the cells were further incubated for $24 \mathrm{~h}$ before experiments under normal growth conditions. The expression of ClC-2 was detected by Western blotting and RT-qPCR analysis.

\section{Cell Proliferation Assay (CCK-8 Assay)}

HConFs were plated at a density of 5,000 cells/well in a 96-well plate. After treatment, $10 \mu \mathrm{L}$ of CCK- 8 solution (BestBio, Jiangsu, China) was added to each well, followed by incubation for another $3 \mathrm{~h}$ at $37^{\circ} \mathrm{C}$. Absorbance at $450 \mathrm{~nm}$ was measured using an automated microplate reader (Model 3001-1387; Thermo Fisher, Waltham, MA, USA). Each group was provided with 6 duplicate holes, and the experiment was repeated 3 times.

\section{Cell Cycle Analysis}

Cell cycle status was assessed by flow cytometry using a Cell Cycle and Apoptosis Analysis Kit (Beyotime, Jiangsu, China). Briefly, cells were grown in 100-mm plates with $10 \%$ FBS for $24 \mathrm{~h}$. After treating the cells with media containing different reagents for $48 \mathrm{~h}$, they were collected and fixed in $75 \%$ ethanol for $24 \mathrm{~h}$ at $4^{\circ} \mathrm{C}$. After rinsing cells with PBS, the cells were stained with PI buffer (containing $500 \mu \mathrm{L}$ staining buffer, $25 \mu \mathrm{L}$ of $20 \times$ propidium iodide, and $10 \mu \mathrm{L}$ of $50 \times$ RNase). The cell cycle distribution was assessed by flow cytometry (CYTOMICS FV 500, Beckman Coulter, Brea, CA, USA).

\section{Reverse Transcription Quantitative Polymerase Chain Reaction}

Total RNA was extracted from HConFs at $60-80 \%$ confluency with RNAiso Plus (Takara Bio Inc., Shiga, Japan), and 500 ng RNA was used to synthesize single-stranded cDNA with the RT-PCR kit (Takara Bio Inc.). Quantitative real-time PCR was performed using methods similar to those previously described [11]. Oligonucleotide primers for the ClC-2, collagen 1, collagen 3, MMP-2, MMP-9, TIMP1, and GAPDH genes were designed using the Primer3 program (http://frodo.wi.mit.edu/primer3/), and sequences are listed in Table 1. 
Table 1. Oligonucleotide primers for genes in quantitative real-time RT-PCR

\begin{tabular}{|c|c|c|}
\hline Human genes & Sequences & Product size, $\mathrm{kb}$ \\
\hline \multirow[t]{2}{*}{$\mathrm{ClC}-2$} & Forward: (5-3') CCCTGGTCATCTTCATTCTCA & \multirow[t]{2}{*}{124} \\
\hline & Reverse: (5-3') TAGGTGCTGCTGTCCGTATG & \\
\hline \multirow[t]{2}{*}{ Collagen 1} & Forward: $\left(5-3^{\prime}\right)$ TCCTCTTTAGCACCCTTTCG & \multirow[t]{2}{*}{128} \\
\hline & Reverse: (5-3') GGACCAGCAACACCATCTG & \\
\hline \multirow[t]{2}{*}{ Collagen 3} & Forward: (5-3') TCAGGGTGTCAAGGGTGAA & \multirow[t]{2}{*}{130} \\
\hline & Reverse: (5-3') AGGGTTTCCATCTCTTCCA & \\
\hline \multirow[t]{2}{*}{ MMP-2 } & Forward: (5-3') TATGGCTTCTGCCCTGAGAC & \multirow[t]{2}{*}{142} \\
\hline & Reverse: (5-3') CACACCACATCTTTCCGTCA & \\
\hline \multirow[t]{2}{*}{ MMP-9 } & Forward: (5-3') AGTCCACCCTTGTGCTCTTC & \multirow[t]{2}{*}{117} \\
\hline & Reverse: (5-3') ACTCTCCACGCATCTCTGC & \\
\hline \multirow[t]{2}{*}{ TIMP1 } & Forward: (5-3') CTGTTGTTGCTGTGGCTGAT & \multirow[t]{2}{*}{130} \\
\hline & Reverse: (5-3') TCTGGTTGACTTCTGGTGTCC & \\
\hline \multirow[t]{2}{*}{ GAPDH } & Forward: (5-3') CAGGAGGCATTGCTGATGAT & \multirow[t]{2}{*}{126} \\
\hline & Reverse: (5-3') CAGGAGGCATTGCTGATGAT & \\
\hline
\end{tabular}

ClC, chloride channel; ClC-2, chloride channel 2; MMP, metalloproteinase; TIMP, tissue inhibitor of metalloproteinase.

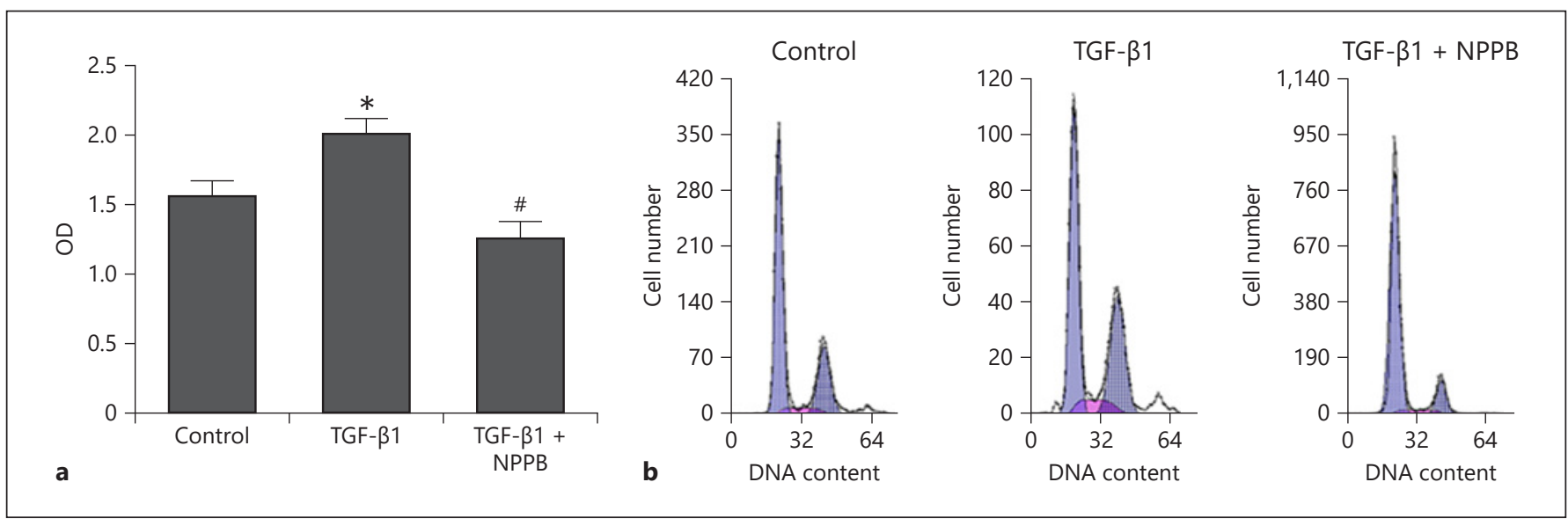

Fig. 1. NPPB inhibits TGF- $\beta 2$-induced HConF cell proliferation and cell cycle progression. a Relative cell counts were determined using the CCK-8 assay and results are expressed as the OD values. Stimulation with $100 \mu \mathrm{M}$ NPPB attenuated TGF- $\beta 1$-induced HConF proliferation. $\mathbf{b}$ Typical cell cycle distribution for HConF cells for various treatments. Results are presented as means $\pm \operatorname{SD}(n=4) ;{ }^{*} p<0.05$, versus control; ${ }^{*} p<0.05$, versus TGF- $\beta 1$ (ANOVA). TGF, transforming growth factor; HConF, human conjunctival fibroblast; OD, optical density.

Western Blotting Analysis

HConFs were washed with precooled PBS 3 times and lysed with RIPA buffer [50 mM Tris ( $\mathrm{pH} 7.4$ ), $150 \mathrm{~mm} \mathrm{NaCl}, 1 \%$ Triton $\mathrm{X}-100,1 \%$ sodium deoxycholate, $0.1 \%$ SDS, and $1 \mathrm{mM}$ PMSF]. The total protein was quantified with the Enhanced BCA Protein Assay Kit (Beyotime). Protein (20 $\mu \mathrm{g}$ per sample) was separated by SDSPAGE and transferred to PVDF membranes, which were then blocked with blocking solution for $1 \mathrm{~h}$ at $37^{\circ} \mathrm{C}$. The membranes were incubated with rabbit polyclonal anti-ClC-2 (Abcam), mouse monoclonal anti-collagen 1 (Abcam), mouse monoclonal anti-collagen 3 (Abcam), rabbit polyclonal anti-MMP-2 (Abcam), rabbit polyclonal anti-MMP-9 (Abcam), rabbit polyclonal anti-TIMP1 (Abcam), and/or mouse monoclonal anti- $\beta$-actin (Beyotime) an- tibodies overnight at $4^{\circ} \mathrm{C}$. Following 2 washes with TBST, the membrane was incubated with horseradish peroxidase-conjugated anti-rabbit IgG secondary antibody (Beyotime) or anti-mouse IgG secondary antibody (Beyotime) for $1 \mathrm{~h}$ at room temperature. Final detection was accomplished with enhanced chemiluminescence Western blotting reagents (Beyotime). Band density was analyzed using ImageJ (NIH, Bethesda, MD, USA).

\section{Assay of Collagen Gel Contraction}

The collagen gel contraction assay was performed according to previously described methods [9], with some modifications. HConFs were harvested by treatment with trypsin-EDTA, washed twice with serum-free MEM, and resuspended in the same 
Table 2. Cell cycle distribution of $\mathrm{HConF}$ in each group (mean $\pm \mathrm{SD}, \%$ )

\begin{tabular}{|c|c|c|c|c|}
\hline \multirow[t]{2}{*}{ Group } & \multirow{2}{*}{$\begin{array}{l}\text { Sample } \\
\text { size }\end{array}$} & \multicolumn{3}{|c|}{ Cell distribution in different cell cycle } \\
\hline & & G0/G1 phase & $S$ phase & $G 2 / M$ phase \\
\hline Control & 4 & $64.43 \pm 3.71$ & $8.37 \pm 3.02$ & $27.20 \pm 1.92$ \\
\hline TGF- $\beta 1$ & 4 & $54.60 \pm 2.19$ & $10.40 \pm 2.19$ & $35.00 \pm 2.56$ \\
\hline TGF- $\beta 1+N P P B$ & 4 & $81.44 \pm 3.14$ & $6.56 \pm 3.02$ & $12.00 \pm 3.23$ \\
\hline$F$ value & & 237.08 & 11.07 & 228.09 \\
\hline$p$ value & & 0.00 & 0.00 & 0.00 \\
\hline
\end{tabular}

TGF, transforming growth factor; HConF, human conjunctival fibroblast.

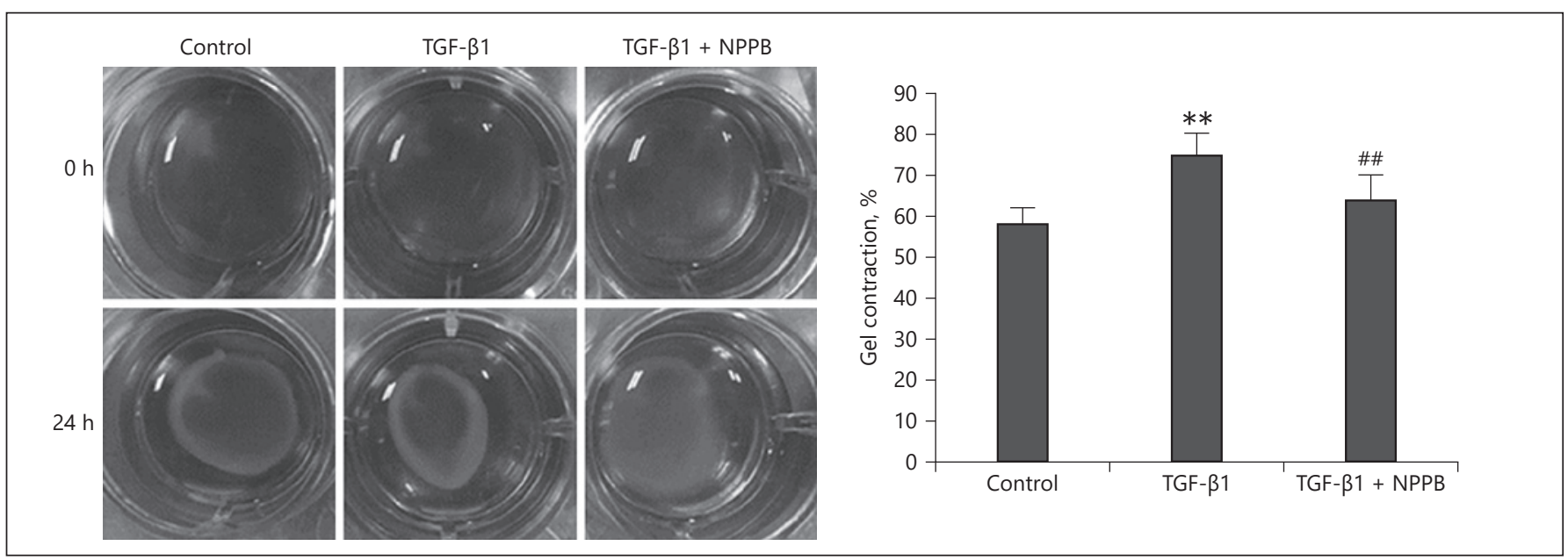

Fig. 2. Effect of NPPB on TGF- $\beta 1$-induced collagen gel contraction mediated by $3 \mathrm{D}$-cultured HConFs. The extent of gel contraction was determined. Results are presented as means $\pm \mathrm{SD}(n=4),{ }^{* *} p<0.01$ versus control; ${ }^{\# \#} p<$ 0.01 versus TGF- $\beta 1$ (ANOVA). TGF, transforming growth factor; HConFs, human conjunctival fibroblasts.

medium. Briefly, collagen 1 (from a rat tail) stock solution ( $5 \mathrm{mg}$ / $\mathrm{mL}$ ) (Solarbio, Beijing, China), $0.1 \mathrm{M} \mathrm{NaOH}, 10 \times$ concentrated PBS, and the cell suspension were mixed on ice at a volume ratio of 200:12:23:765 (final concentration of type 1 collagen, $1 \mathrm{mg} / \mathrm{mL}$ and final cell density, $\left.2 \times 10^{5} / \mathrm{mL}\right)$. A portion $(0.5 \mathrm{~mL})$ of the mixture was added to each well of the 24-well culture plate and allowed to solidify by incubation at $37^{\circ} \mathrm{C}$ under $5 \% \mathrm{CO}_{2}$ for $20 \mathrm{~min}$. The collagen gels were freed from the sides of the wells using a $10-\mu \mathrm{L}$ tip, and serum-free MEM $(0.5 \mathrm{~mL})$ containing TGF- $\beta 1(10 \mathrm{ng} / \mathrm{mL})$ and/or NPPB was then added to the top of each gel. The gel area was examined at $48 \mathrm{~h}$ using ImageJ. For normalization, the area of the collagen gel containing untreated $\mathrm{HConFs}$ was set to $100 \%$, and the fold changes in the area for each treatment group were determined. Four gels were assayed for each experimental condition, and all experiments were repeated 4 times.

\section{Data Analyses}

Results are expressed as means \pm standard error (number of observations), and differences among groups were evaluated using ANOVA. Statistical significance was defined as $p<0.05$. All experiments were repeated 4 times.

\section{Results}

\section{Effect of NPPB on TGF- $\beta 1$-Induced HConF}

Proliferation and Cell Cycle

As shown in Figure 1a, TGF- $\beta 1(10 \mathrm{ng} / \mathrm{mL})$ increased HConF proliferation by $28.66 \%$ ( $p<0.05$ vs. control) after $48 \mathrm{~h}$ of stimulation. HConFs treated with NPPB $(100 \mu \mathrm{M})$ in the presence of TGF- $\beta 1$ showed a $37.62 \%$ ( $p<0.05 \mathrm{vs.}$ control) reduction in cell viability compared to TGF- $\beta 1$ treated cells. These results demonstrate that NPPB inhibits TGF- $\beta 1$-induced HConF cell proliferation.

There were statistically significant differences in the proportion of cells in each cell cycle among the 3 groups (G0/G1 phase: $F=237.08, p=0.00$; $S$ phase: $F=11.07, p=$ 0.00 ; and $G 2 / M$ phase: $F=228.09, p=0.00$ ). Compared with the control group and TGF- $\beta 1+$ NPPB group, the proportion of cells in the $G 1$ phase was significantly reduced in the TGF- $\beta 1$ treatment group, while the propor- 


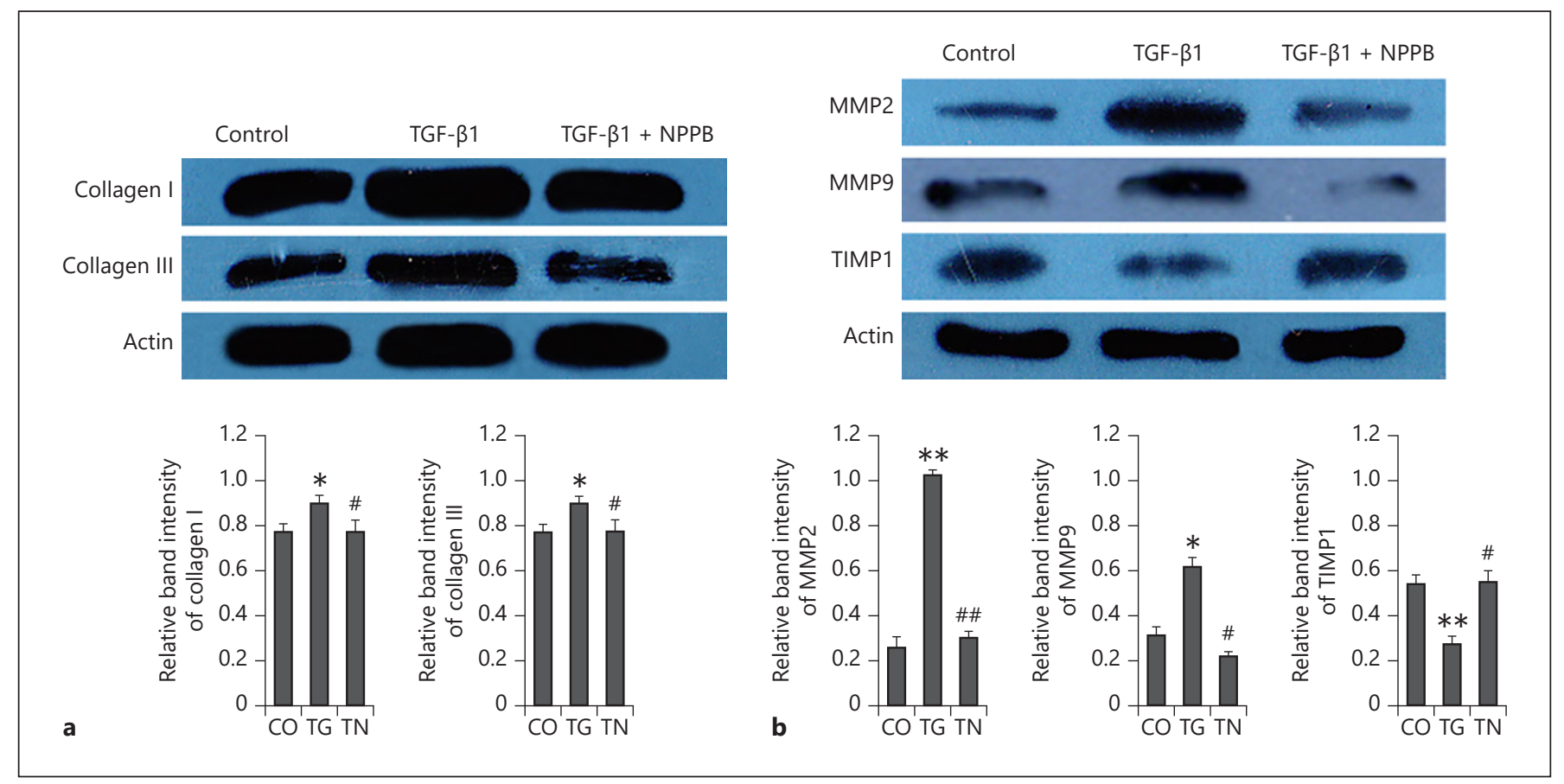

Fig. 3. Protein and mRNA expression of collagen 1, collagen 3, MMP-2, MMP-9, and TIMP1 in HConFs after different treatments as determined by Western blotting and RT-PCR. a Protein expression of collagen 1 and collagen 3 . b Protein expression of MMP-2, MMP-9, and TIMP1. c mRNA expression of collagen 1, collagen 3, MMP-2, MMP-9, and TIMP1. Results are presented as means \pm SD $(n=4) ;{ }^{*} p<0.05,{ }^{* *} p<0.01$ versus control; ${ }^{*} p<0.05$,

tion of cells in the $S$ phase and $G 2 / M$ phase was increased with statistically significant differences. This result showed that TGF- $\beta 1$ promoted cell cycle progression by advancing cells from the $G 0 / G 1$ to $S$ and $G 2 / M$ phases, but NPPB inhibited TGF- $\beta 1$-induced cell cycle progression by arresting cells in the $G 0 / G 1$ phases (Fig. 1b; Table 2).

\section{Effect of NPPB on TGF- $\beta 1$-Induced Collagen Gel Contraction Mediated by HConFs}

As shown in Figure 2, exposure to TGF- $\beta 1$ stimulated HConF-mediated collagen contraction of the free-floating three-dimensional collagen gel and resulted in a significant reduction in the gel area as compared to that of controls $(p<0.01)$. The gel area of the TGF- $\beta 1+$ NPPB group was larger than the TGF- $\beta 1$ group $(p<0.01)$.

\section{Effect of NPPB on TGF- $\beta 1$-Induced Collagen Synthesis and $M M P / T I M P$ Expression}

TGF- $\beta 1$ induced high levels of collagen 1 and collagen 3 expressions at the protein and mRNA levels in HConFs compared to levels in control cells. NPPB significantly at-
${ }^{\# \#} p<0.01$ versus TGF- $\beta 1$. CO, control; TG, TGF- $\beta 1$ (HConFs treated with $10 \mathrm{ng} / \mathrm{mL}$ TGF- $\beta 1$ for $48 \mathrm{~h}$ ); TN, TGF- $\beta 1+$ NPPB (HConFs treated with $10 \mathrm{ng} / \mathrm{mL}$ TGF- $\beta 1$ and $100 \mu \mathrm{M}$ NPPB for 48 h); TGF, transforming growth factor; HConF, human conjunctival fibroblast; MMP, metalloproteinase; TIMP, tissue inhibitor of metalloproteinase. tenuated the TGF- $\beta 1$-induced expression of collagen 1 and collagen 3 (Fig. $3 \mathrm{a}$ ).

TGF- $\beta 1$ increased the protein and mRNA expression levels of MMP-2 and MMP-9, which negatively regulate collagen synthesis and deposition. NPPB significantly decreased the TGF- $\beta 1$-induced increases in MMP- 2 and MMP-9. TGF- $\beta 1$ resulted in lower expression of TIMP1, a positive regulator that promotes collagen expression, at the protein and mRNA levels in HConFs than in control cells. NPPB significantly attenuated the TGF- $\beta 1$-induced reduction in TIMP1 expression (Fig. 3b).

\section{Effect of ClC-2 siRNA on TGF- $\beta 1$-Induced}

\section{Proliferation and Cell Cycle in HConF}

$\mathrm{HConF}$ transfected with ClC-2 siRNA in the presence of TGF- $\beta 1$ showed a $39.20 \%$ reduction in cell viability compared to that of TGF- $\beta 1$-treated negative siRNA control cells. Nontransfected and mutant siRNA-transfected cells had similar viabilities (Fig. 4a). These results suggest that the knockout of $\mathrm{ClC}-2$ inhibits TGF- $\beta 1$-induced HConF cell proliferation. 


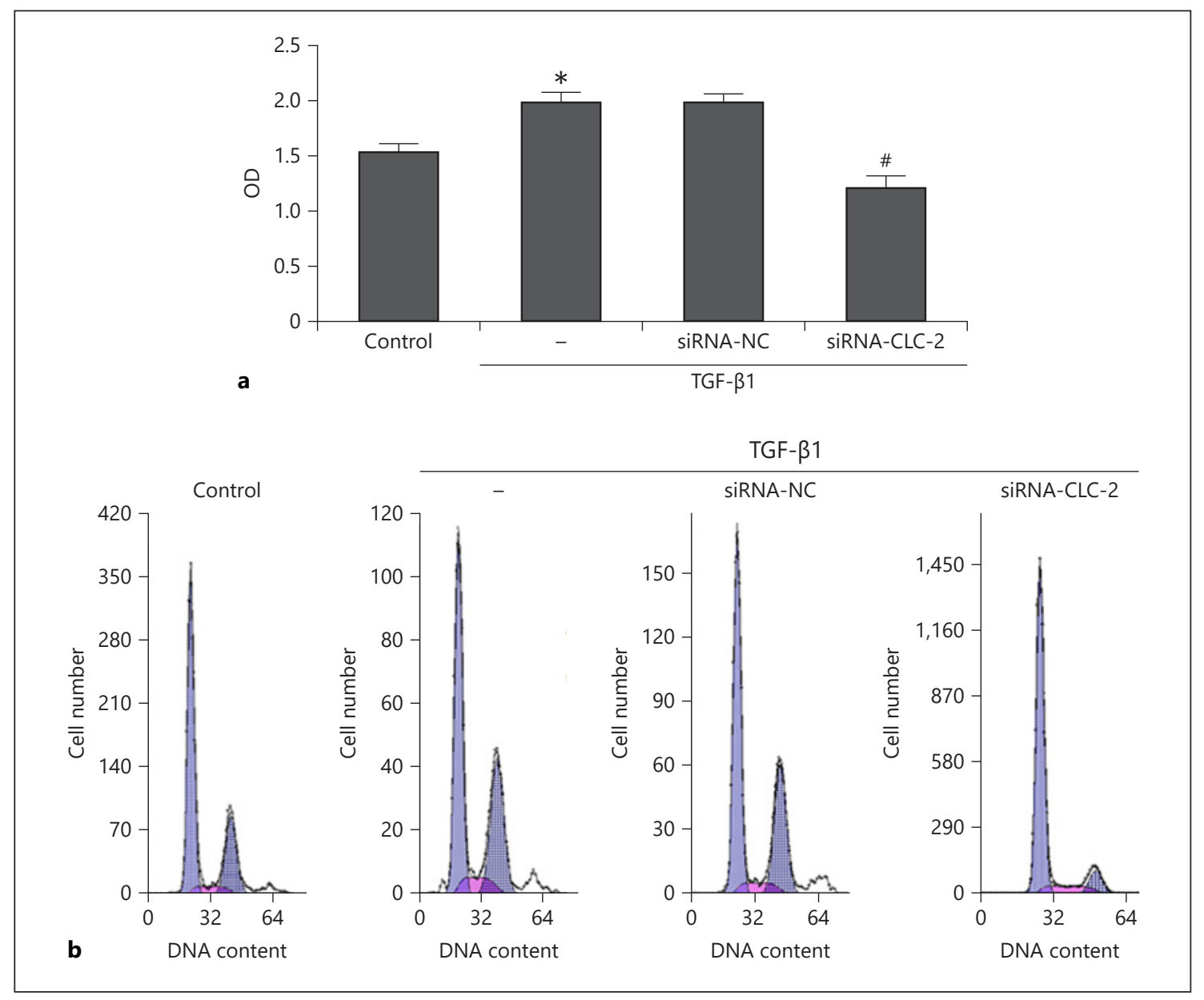

Fig. 4. a Relative cell counts were detected using the CCK- 8 assay and results are expressed as OD values. b Effects of ClC-2 siRNA on $\mathrm{HConF}$ cell cycle progression, as determined by flow cytometry. Typical cell cycle distribution and quantitative distribution of cells in different phases for various treatments. Results are presented as

The proportion of cells in the $G 0 / G 1$ phase was greater in the TGF- $\beta 1+$ ClC- 2 siRNA group than in the TGF- $\beta 1$ group and TGF- $\beta 1+$ negative siRNA control group, but the proportions in $S$ and $G 2 / M$ phases were lower in the TGF- $\beta 1+C l C-2$ siRNA group than the TGF- $\beta 1$ group and the TGF- $\beta 1+$ negative siRNA control group (Fig. 4b). TGF- $\beta 1$ promoted cell cycle progression, whereas knockout of ClC- 2 inhibited TGF- $\beta 1$-induced cell cycle progression by arresting the cells in the $G 0 / G 1$ phases.

\section{Effect of ClC-2 siRNA on TGF- $\beta 1$-Induced Collagen Gel Contraction Mediated by $\mathrm{HConF}$}

The gel area of the TGF- $\beta 1+$ ClC- 2 siRNA group was larger than the TGF- $\beta 1$ group and TGF- $\beta 1$-treated negative siRNA control group. The TGF- $\beta 1$-induced contrac- means \pm SD $(n=4) ;{ }^{*} p<0.05$, versus control; ${ }^{*} p<0.05$, versus TGF- $\beta 1$ (ANOVA). TGF, transforming growth factor; HConF, human conjunctival fibroblast; $\mathrm{OD}$, optical density; $\mathrm{ClC}$, chloride channel; ClC-2, chloride channel 2.

tion of HConF cells was inhibited by the siRNA-mediated downregulation of ClC-2 (Fig. 5).

\section{Effect of ClC-2 siRNA on TGF- $\beta 1$-Induced Collagen} Synthesis and MMP/TIMP Expression

As shown in Figure $6 \mathrm{a}$ and $\mathrm{c}$, the knockout of ClC-2 significantly inhibited the TGF- $\beta 1$-induced protein and mRNA expression of collagen 1 and collagen 3 . The protein and mRNA expression levels of collagen 1 and collagen 3 were similar in TGF- $\beta 1$-treated nontransfected cells and TGF- $\beta 1$-treated negative siRNA control cells.

The protein and mRNA expression levels of MMP-2 and MMP-9 were lower in the ClC-2 siRNA-transfected group than in the nontransfected and mutant siRNAtransfected groups in the presence of TGF- $\beta 1$. Converse- 
Fig. 5. Effect of ClC-2 siRNA on TGF- $\beta 1$ induced collagen gel contraction mediated by $3 \mathrm{D}$-cultured HConFs. The extent of gel contraction was determined at 0 and $24 \mathrm{~h}$. Results are presented as means \pm SD $(n=$ $4) ;{ }^{* *} p<0.01$ versus control; ${ }^{\# \#} p<0.01$ versus TGF- $\beta 1$. TGF, transforming growth factor; HConF, human conjunctival fibroblast; $\mathrm{ClC}$, chloride channel; ClC-2, chloride channel 2.
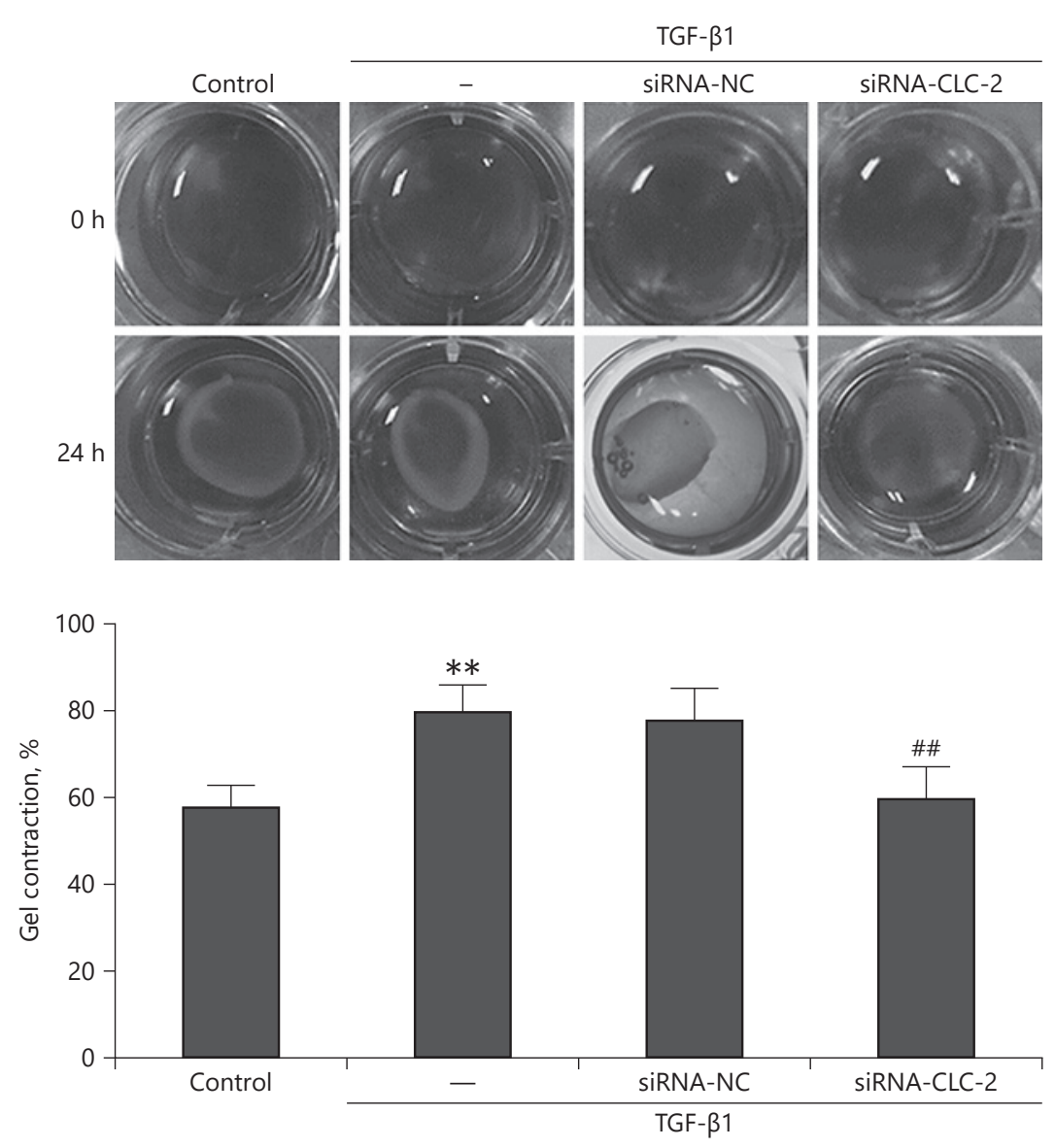

ly, the protein and mRNA expression of TIMP1 was higher in the ClC-2 siRNA-transfected group than in the nontransfected and mutant siRNA-transfected groups in the presence of TGF- $\beta 1$ (Fig. $6 \mathrm{~b}, \mathrm{c}$ ).

\section{Discussion/Conclusion}

In the present study, we induced a series of conjunctival scarring responses with TGF- $\beta 1$. Subsequently, the effects of the ClC blocker NPPB and ClC-2 ClC on HConF scarring responses were measured. We found that NPPB and ClC-2 siRNA transfection inhibited TGF- $\beta 1$-induced HConF proliferation, collagen synthesis, and collagen gel contraction. Exaggerated wound contraction induced by elevated TGF- $\beta$ levels has become a therapeutic target in fibrosis [12]. A study by Arslan et al. [13] showed that filtration surgeries increased TGF- $\beta$ expression. Similar to our results, Liu et al. [14] found that TGF- $\beta$-induced collagen gel contraction was mediated by human Tenon fibroblasts.
$\mathrm{ClCs}$ are expressed in nearly all eukaryotic cells and play an important role in cell proliferation, apoptosis, and migration [15]. The regulatory effect of $\mathrm{ClCs}$ on cell proliferation has been shown in many studies [16]. A study by Warsi et al. [17] showed that downregulation of the $\mathrm{ClC} \mathrm{ClC}-2$ by Janus kinase 3 influenced cell proliferation and apoptosis. In osteosarcoma cells, ClC-3 silencing inhibited cell proliferation and migration via the AKT/GSK3 $\beta$ signaling pathway [18]. Li et al. [19] found that overexpression of the chloride intracellular channel 5 inhibited proliferation of $\mathrm{C} 2 \mathrm{C} 12$ cells and drove more $\mathrm{C} 2 \mathrm{C} 12$ cells into the $G 0 / G 1$ phase. We previously blocked the $\mathrm{ClC}$ of ARPE19 in human adult RPE cells using NPPB and tamoxifen and found that this inhibited proliferation by arresting cells in the G0/G1 phase [20]. ClC-2, a ubiquitously expressed member of the superfamily of $\mathrm{ClC}$ transporters and channels, has been confirmed to be expressed in human conjunctival fibroblasts [11]. Our result showed that NPPB and ClC-2 siRNA transfection inhibited 


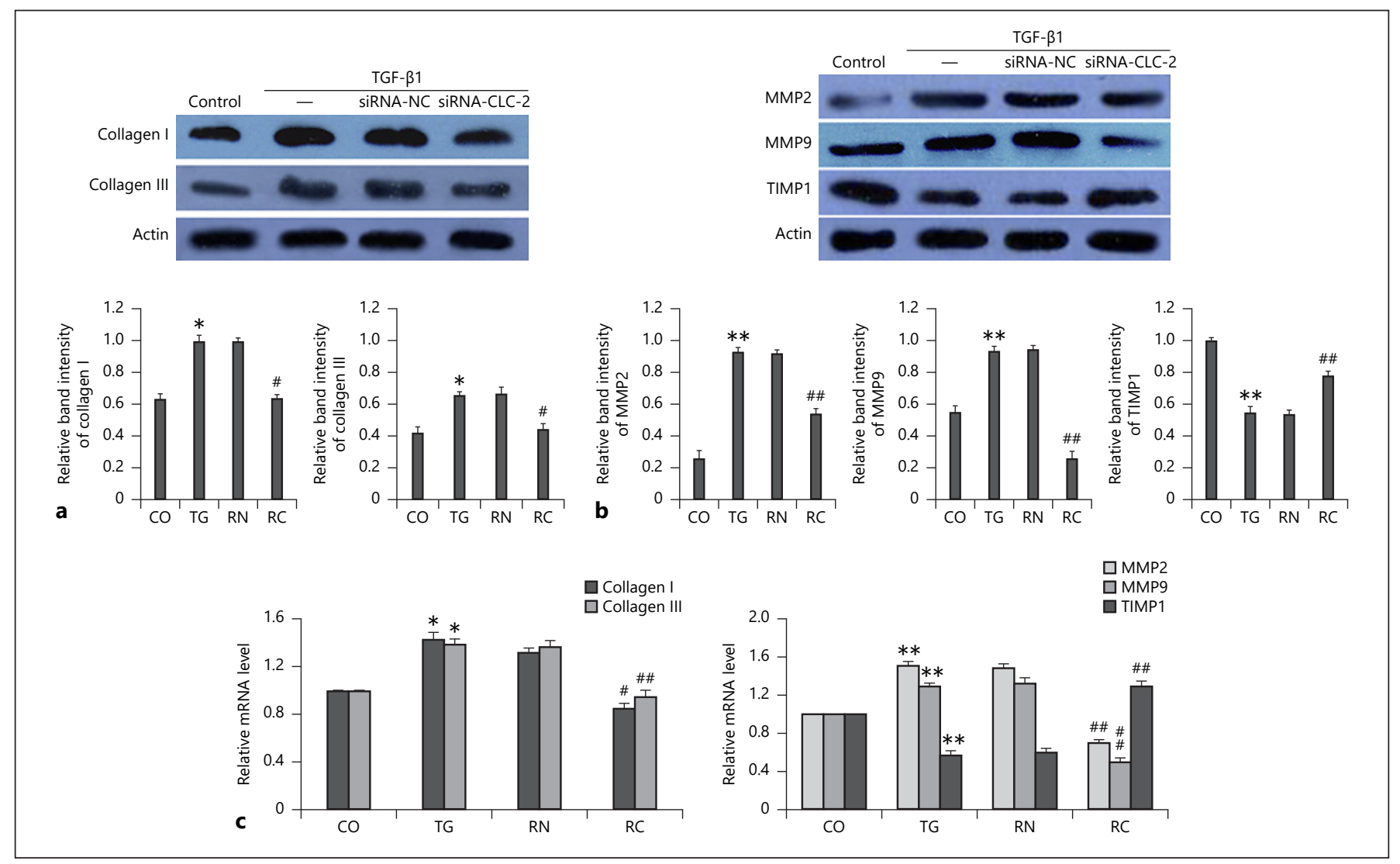

Fig. 6. Protein and mRNA expression of collagen 1, collagen 3, MMP-2, MMP-9, and TIMP1 in HConFs after different treatments as determined by Western blotting and RT-PCR. a Protein expression of collagen 1 and collagen 3 . b Protein expression of MMP-2, MMP-9, and TIMP1. c mRNA expression of collagen 1, collagen 3, MMP-2, MMP-9, and TIMP1. Results are presented as means \pm SD $(n=4) ;{ }^{*} p<0.05,{ }^{* *} p<0.01$ versus control; ${ }^{*} p<0.05$, ${ }^{\# \#} p<0.01$ versus TGF- $\beta 1$. CO, control; TG, TGF- $\beta 1$ (HConFs

TGF- $\beta 1$-induced HConF cell proliferation by arresting cells in the $G 0 / G 1$ phase.

Recent studies have highlighted the role of ClCs in wound healing. Fuchigami et al. [21] showed that the $\mathrm{ClC}$ blocker NPPB reduced epidermal wound closure by blocking the $\mathrm{ClC}-3 \mathrm{ClC}$. Further, local enhancement of ClC-3 expression at the leading edge of the wounded epidermis was specific to closing wounds, suggesting that the intracellular translocation of $\mathrm{ClC}-3$ was involved in wound closure. Schiller et al. [22] found that CFTR ClCs positively regulated wound healing in cell culture models of the airway surface epithelium. Thus, inhibition of conjunctival fibroblast proliferation is the main strategy for treatment of antiglaucoma filtration scarring. treated with $10 \mathrm{ng} / \mathrm{mL}$ TGF- $\beta 1$ for $48 \mathrm{~h}$ ); RN, TGF- $\beta 1+$ siRNANC (HConFs treated with $10 \mathrm{ng} / \mathrm{mL}$ TGF- $\beta 1$ and transfected with 80 nM mutant ClC-2 siRNA); RC, TGF- $\beta 1+$ ClC- 2 siRNA (HConFs treated with $10 \mathrm{ng} / \mathrm{mL}$ TGF- $\beta 1$ and transfected with $80 \mathrm{nM} \mathrm{ClC}-2$ siRNA); TGF, transforming growth factor; HConF, human conjunctival fibroblast; $\mathrm{ClC}$, chloride channel; $\mathrm{ClC}-2$, chloride channel 2; MMP, metalloproteinase; TIMP, tissue inhibitor of metalloproteinase.

Cell-mediated tissue contraction is a complex process that involves various cellular activities, including cell migration and reorganization of the ECM, and MMPs have been implicated in wound contraction and matrix reorganization [23]. Collagen contraction involves the cell-directed reorganization of collagen fibrils and plays a significant role in wound healing. In the present study, a three-dimensional model of wound healing that closely mimics the in vivo situation was established. We found that TGF- $\beta 1$ promoted HConF collagen synthesis and collagen gel contraction. NPPB inhibited TGF- $\beta 1$-induced cell collagen synthesis and collagen gel contraction mediated by $\mathrm{HConFs}$, and $\mathrm{ClC}-2$ gene knockout inhibited TGF- $\beta 1$-induced HConF collagen synthesis and collagen gel contraction. Multiple studies have shown that chloride 
ClCs are involved in collagen synthesis [24-26]. Qi et al. [24] showed that the ClC blocker DIDS inhibited the collagen release of HFL1 fibroblasts. Downregulation of CLIC4 also reduced TGF- $\beta 1$-induced expression of ECM components in primary fibroblasts [25]. In the development of atrial fibrillation, CLIC 1,4 , and 5 played an important role by interacting with collagen $4[26,27]$.

Inhibition of MMPs has been shown to limit subconjunctival scarring after experimental glaucoma filtration surgery in rabbits [27]. The balance between MMPs and TIMPs in the ECM is important for regulation of the ECM and wound healing [23]. The ratio of MMPs to TIMPs is an important factor in various biological activities, including cell migration, angiogenesis, and remodeling of the ECM [28]. Du and Yang [21] found that ClC-3 knockout attenuated osteosarcoma cell migration via downregulation of MMP-2 and MMP-9. In the present study, TGF- $\beta 1$ induced expression of MMP- 2 and MMP9 but inhibited TIMP1 expression in HConFs. TGF- $\beta 1$ induced expression of MMP-2 and MMP-9 was inhibited by NPPB and ClC-2 siRNA transfection in HConFs. NPPB and ClC-2 siRNA transfection increased TIMP1 expression in the presence of TGF- $\beta 1$.

Consistent with our results, Guan et al. [29] found that MMP-1, MMP-2, and MMP-3 levels were increased by TGF- $\beta 1$, while TGF- $\beta 1$ inhibited TIMP1 expression. They also found knockdown of ClC-3 expression inhibited MMP-9 expression in ectopic endometrial cells. Wang et al. [30] silenced the $\mathrm{ClC}-3$ gene and found that MMP-3 and MMP-9 levels were reduced. Our results showed that the ClC-2 knockout decreased TGF- $\beta 1$ induced proliferation, collagen synthesis, and contractility of HConFs cultured in a three-dimensional collagen gel, in part by blocking MMP production. Further in vivo studies are needed to confirm the preventive effect of NPPB and ClC-2 siRNA on filtering bleb scarring after filtration surgery.

\section{Acknowledgements}

This work was supported by Yanbian University Postdoctoral Workstation. The authors would like to thank Editage for English language editing.

\section{Statement of Ethics}

This research was conducted ethically in accordance with the World Medical Association Declaration of Helsinki.

\section{Conflict of Interest Statement}

The authors have no conflict of interest to declare.

\section{Funding Sources}

This work was supported by the National Natural Science Foundation of China Project (81760171) and Jilin Province Education Department "13th Five-Year" Science and Technology Project (JJKH20191117KJ).

\section{Author Contributions}

Lixia Sun wrote the article; Renzhe Cui conducted the experiment; Huan Meng guided the experiment; Xiwen Liu conducted the experiment; Xin Liu conducted the experiment; Yan Lu conducted the experiment; Kun Liu conducted the experiment; Liang Jia conducted the experiment; and Yajuan Zheng designed the project.

\section{References}

1 Merkel PA, Silliman NP, Clements PJ, Denton CP, Furst DE, Mayes MD, et al. Patterns and predictors of change in outcome measures in clinical trials in scleroderma: an individual patient meta-analysis of 629 subjects with diffuse cutaneous systemic sclerosis. Arthritis Rheum. 2012 Oct;64(10):3420-9.

2 Hoogewerf CJ, van Baar ME, Middelkoop E, van Loey NE. Patient reported facial scar assessment: directions for the professional. Burns. 2014 Mar;40(2):347-53.

3 Chen N, Guo D, Guo Y, Sun Y, Bi H, Ma X. Paclitaxel inhibits cell proliferation and collagen lattice contraction via TGF- $\beta$ signaling pathway in human tenon's fibroblasts in vitro. Eur J Pharmacol. 2016 Apr 15;777: 33-40.
4 Meyer-Ter-Vehn T, Grehn F, Schlunck G. Localization of TGF-beta type 2 receptor and ED-A fibronectin in normal conjunctiva and failed filtering blebs. Mol Vis. 2008 Jan 25;14: 136-41.

5 Valimaki J, Uusitalo H. Matrix metalloproteinases (MMP-1, MMP-2, MMP-3 and MMP-9, and TIMP-1, TIMP-2 and TIMP-3) and markers for vascularization in functioning and non-functioning bleb capsules of glaucoma drainage implants. Acta Ophthalmol. 2015 Aug;93(5):450-6.

6 Strange K. Of mice and worms: novel insights into ClC-2 anion channel physiology. News Physiol Sci. 2002 Feb;17:11-6.

7 Grunder S, Thiemann A, Pusch M, Jentsch TJ. Regions involved in the opening of CIC-2 chloride channel by voltage and cell volume. Nature. 1992 Dec 24-31;360(6406):759-62.

8 Bosl MR, Stein V, Hubner C, Zdebik AA, Jordt SE, Mukhopadhyay AK, et al. Male germ cells and photoreceptors, both dependent on close cell-cell interactions, degenerate upon ClC-2 $\mathrm{Cl}(-)$ channel disruption. EMBO J. 2001 Mar 15;20(6):1289-99.

9 Lu J, Xu F, Zhang Y, Lu H, Zhang J. ClC-2 knockdown prevents cerebrovascular remodeling via inhibition of the $\mathrm{Wnt} / \beta$-catenin signaling pathway. Cell Mol Biol Lett. 2018;23:29.

10 Pan F, Guo R, Cheng W, Chai L, Wang W, $\mathrm{Cao} \mathrm{C}$, et al. High glucose inhibits ClC-2 chloride channels and attenuates cell migration of rat keratinocytes. Drug Des Devel Ther. 2015; 9:4779-91.
Role of Chloride Channel in Glaucoma Bleb Scarring
Ophthalmic Res 2021;64:775-784 DOI: $10.1159 / 000507632$ 
11 Sun L, Dong Y, Zhao J, Yin Y, Zheng Y. The CLC-2 chloride channel modulates ECM synthesis, differentiation, and migration of human conjunctival fibroblasts via the PI3K/Akt signaling pathway. Int J Mol Sci. 2016 Jun 9; 17(6).

12 Kasuya A, Tokura Y. Attempts to accelerate wound healing. J Dermatol Sci. 2014 Dec; 76(3):169-72.

13 Arslan S, Aydemir O, Güler M, Dağlı AF. Modulation of postoperative scarring with tacrolimus and octreotide in experimental glaucoma filtration surgery. Curr Eye Res. 2012 Mar;37(3):228-33.

14 Liu Y, Kimura K, Orita T, Teranishi S, Suzuki $\mathrm{K}$, Sonoda KH. Inhibition by all-trans-retinoic acid of transforming growth factor- $\beta$ induced collagen gel contraction mediated by human tenon fibroblasts. Invest Ophthalmol Vis Sci. 2014 Jun 3;55(7):4199-205.

15 Okada Y, Maeno E. Apoptosis, cell volume regulation and volume-regulatory chloride channels. Comp Biochem Physiol, Part A Mol Integr Physiol. 2001 Oct;130(3):377-83.

16 Zhou F-M, Huang Y-Y, Tian T, Li X-Y, Tang Y-B. Knockdown of chloride channel-3 inhibits breast cancer growth in vitro and in vivo. J Breast Cancer. 2018 Jun;21(2):103-11.

17 Warsi J, Elvira B, Hosseinzadeh Z, Shumilina E, Lang F. Downregulation of chloride channel ClC-2 by Janus kinase 3. J Membr Biol. 2014 May;247(5):387-93.
18 Du S, Yang L. ClC-3 chloride channel modulates the proliferation and migration of osteosarcoma cells via AKT/GSK3 $\beta$ signaling pathway. Int J Clin Exp Pathol. 2015;8(2):162230.

19 Li F, Yin J, Yue T, Liu L, Zhang H. The CLIC5 (chloride intracellular channel 5) involved in C2C12 myoblasts proliferation and differentiation. Cell Biol Int. 2010 Mar 8;34(4):37984.

20 Zhao J, Zhong W, Sun L, Yin Y, Zheng Y. Effect of chloride channel activity on retinal pigment cell proliferation and migration. Mol Med Rep. 2017 Apr;15(4):1771-6.

21 Fuchigami T, Matsuzaki T, Ihara S. Possible roles of $\mathrm{ENaC}$ and $\mathrm{Cl}(-)$ channel in wound closure in Xenopus laevis embryos. Zool Sci. 2011 Oct;28(10):703-11.

22 Schiller KR, Maniak PJ, O’Grady SM. Cystic fibrosis transmembrane conductance regulator is involved in airway epithelial wound repair. Am J Physiol, Cell Physiol. 2010 Nov; 299(5):C912-21.

23 Ito T, Ohguro H, Mamiya K, Ohguro I, Nakazawa M. Effects of antiglaucoma drops on MMP and TIMP balance in conjunctival and subconjunctival tissue. Invest Ophthalmol Vis Sci. 2006 Mar;47(3):823-30.

24 Qi S, den Hartog GJ, Bast A. Superoxide radicals increase transforming growth factor-beta 1 and collagen release from human lung fibroblasts via cellular influx through chloride channels. Toxicol Appl Pharmacol. 2009 May $15 ; 237(1): 111-8$
25 Shukla A, Edwards R, Yang Y, Hahn A, Folkers K, Ding J, et al. CLIC4 regulates TGF- $\beta$ dependent myofibroblast differentiation to produce a cancer stroma. Oncogene. 2014 Feb 13;33(7):842-50.

26 Jiang YY, Hou HT, Yang Q, Liu XC, He GW. Chloride channels are involved in the development of atrial fibrillation - a transcriptomic and proteomic study. Sci Rep. 2017 Aug 31; 7(1):10215.

27 Fingleton B. Matrix metalloproteinases as regulators of inflammatory processes. Biochim Biophys Acta. 2017 Nov;1864(11 Pt A): 2036-42. A):

28 Wong TT, Mead AL, Khaw PT. Matrix metalloproteinase inhibition modulates postoperative scarring after experimental glaucoma filtration surgery. Invest Ophthalmol Vis Sci. 2003 Mar;44(3):1097-103.

29 Guan YY, Wang GL, Zhou JG. The ClC-3 Clchannel in cell volume regulation, proliferation and apoptosis in vascular smooth muscle cells. Trends Pharmacol Sci. 2006 Jun;27(6): 290-6.

30 Wang B, Xie J, He HY, Huang EW, Cao QH, Luo L, et al. Suppression of CLC-3 chloride channel reduces the aggressiveness of glioma through inhibiting nuclear factor- $\mathrm{\kappa B}$ pathway. Oncotarget. 2017 Sep 8;8(38):63788-98. 